\title{
The importance of lipid spectrum changes in women with polycystic ovary syndrome (PCOS)
}

\author{
Sarapatkova $\mathrm{H}^{1,2}$, Sarapatka $\mathrm{J}^{1}$, Frysak $\mathrm{Z}^{2}$ \\ Endocrinology and Internal Outpatient Department, Olomouc, Czech Republic. h.sarapatkova@atlas.cz
}

\begin{abstract}
Aim of the study was to compare levels of blood lipids, anthropometric characteristics and their relationship in women with PCOS with a group of healthy women.

Methods and results: We determined anthropometric indicators (BMI - body mass index, waist measurement, waist/hip ratio), blood lipid levels (total cholesterol $\mathrm{TCH}$, high density cholesterol $\mathrm{HDL}$, low density cholesterol LDL and triglycerides TAG) in a group of women with PCOS and a healthy control group.

Levels of evaluated blood lipids in women with PCOS and the healthy control group were within the normal range. Women with PCOS differed statistically significantly from the control group in a higher concentration of triglycerides (control $1.01 \pm 0.7 \mathrm{mmol}^{-1}{ }^{-1}$, PCOS $1.17 \pm 0.58$ ). In women with PCOS we proved a positive correlation between BMI, total cholesterol, LDL cholesterol and levels of triglycerides. In women with PCOS waist measurement showed a statistically significant relationship to values of LDL cholesterol, HDL cholesterol and level of triglycerides. Conclusions: All women with PCOS in our study had very good levels of blood lipoproteins (total cholesterol, LDL and HDL cholesterol, triglycerides) so we are not sure if there is any importance of changes in blood lipid metabolism in these patients (Tab. 1, Fig. 2, Ref. 16). Text in PDF www.elis.sk.

Key words: polycystic ovary syndrome, total cholesterol, HDL cholesterol, LDL cholesterol, triglycerides, anthropometric characteristics.
\end{abstract}

With its incidence of about $6-8 \%$, the polycystic ovary syndrome (PCOS) is one of the most common endocrine illnesses in women of fertile age $(1,2)$. It is characterised by hyperandrogenism, oligo/anovulation, presence of polycystic ovaries despite the elimination of other conditions with overproduction of androgens $(3,4)$. Despite the fact that more than 70 years have passed since its summary definition, PCOS is still an illness of uncertain etiology and pathogenesis (5).

Affected women have a higher probability of infertility, more frequent miscarriages, and certain metabolic changes with an increased risk of glucose-tolerance disorder, occurrence of diabetes mellitus type 2, hyperlipidemia, obesity, and arterial hypertension. With regard to cumulation of the stated risk factors, the possibility of a higher risk of cardiovascular illness can be anticipated $(6,7)$. Deficient production of progesterone together with chronic estrogen stimulation is the precondition for endometrial hyperplasia and endometrial cancer.

The clinical, laboratory and morphological manifestations (discovery of polycystic ovaries) in women with PCOS are diverse, ranging from an image of an obese woman with distinct hirsutism and amenorrhea, expressed in metabolic changes (glucose toler-

${ }^{1}$ Endocrinology and Internal Outpatient Department, Olomouc, Czech Republic, and ${ }^{2}$ Department of Internal Medicine III, Nephrology, Rheumatology and Endocrinology, University Hospital, Olomouc, Czech Republic

Address for correspondence: $\mathrm{H}$. Sarapatkova, MD, $\mathrm{PhD}$, Endocrinology and Internal Outpatient Department, Fibichova 9, CZ-772 00 Olomouc, Czech Republic.

Phone: +420.585414620 ance disorder, possible DM, hyperlipidemia), to that of a slim woman with no visible evidence of hirsutism, with oligomenorrhea, who nonetheless becomes spontaneously pregnant $(8,9)$. It can be anticipated that further discoveries in the field of molecular genetics and biology will divide PCOS into several separate categories which will have a mutual clinical resemblance, but will be of different aetiology. The development of the illness is determined by mutual interactions of numerous genetic abnormities and factors of the external environment.

Women with PCOS were expected to have changes in blood lipid metabolism manifested in various types and degrees. Several studies find typical atherogenic dyslipidemia, which can be found in both obese and diabetic patients with increased levels of triglycerides (TAG) and reduced levels of HDL cholesterol; some studies point out increased levels of LDL cholesterol (10) but the results seem to be inconclusive as well as are the views on the cardiovascular risk in these patients.

In our study we focused on changes in levels of blood lipids and their relationship to anthropometric parameters in women with PCOS treated at our clinic. The results obtained were then compared with the control group.

\section{Material and methods}

\section{Patient base}

The observed group comprised women referred for examination at endocrinologic and internal departments in years 2008 2010 due to hirsutism, disorder of menstrual cycle and alopecia. 
All included women fulfilled the criteria for PCOS according to the Rotterdam consensus. There were 72 patients with an average age of 31.6. The control group comprised 43 healthy women without disorder of menstrual cycle, without hirsutism or hyperandrogenism. Both groups comprised only women who were not taking hormonal contraceptives or who had stopped taking them at least three months before, at their own decision.

All the patients consented to undergoing more extensive laboratory investigation, and to the results being used for the purpose of our study.

The women underwent clinical examination including the determination of BMI in $\mathrm{kg} / \mathrm{m}^{2}$, waist measurement in $\mathrm{cm}$ and waist/hip ratio. Blood samples were taken in the morning on an empty stomach in the follicular period of the cycle (2nd - 5th day of menstruation) or during amenorrhea. Levels were determined in the serum for follicle-stimulating hormone (FSH), luteinizing hormone (LH), estradiol, total testosterone and free testosterone, androstenedione, dehydroepiandrosterone sulphate (DHEAS), prolactin, thyroid function (TSH thyroid-stimulating hormone, free thyroxine, autoantibodies against thyroperoxidase and thyroglobulin), level of 17-hydroxyprogesterone, level of total cholesterol, HDL cholesterol, LDL cholesterol and triglycerides, glycaemia (blood sugar) and glycated haemoglobin.

\section{Laboratory analysis}

Tests were performed for total cholesterol, LDL cholesterol, triglycerides and thyroglobulin with the use of Advia 1650 biochemical analyser (Erba Lachema reagents), HDL cholesterol was determined via direct immunoinhibition method. To determine levels of FSH, LH, estradiol, progesterone, prolactin, total testosterone, DHEAS, C peptide, TSH, free thyroxine, autoantibodies against thyroperoxidase and thyroglobulin an Architect analyser was used (Abbott reagents) via chemiluminescent immunoanalysis method on microparticles - CMIA (Chemiluminiscent Microparticle Immunoassay). Free testosterone and androstenedione were determined via the Competitive immunoenzymatic colorimetric method (DiaMetra company); 17-hydroxyprogesterone was determined via ELISA method. Glycated haemoglobin was determined via the turbidimetric immunoinhibition method.

\section{Statistical processing}

Calculations were carried out using statistical systems by StatSoft CR s.r.o. (2007). STATISTICA Cz (a software system for data analysis) version 8.0 www.statsoft.cz and SPSS v.12.0. Non-parametric methods were chosen on the basis of results of the Shapiro-Wilk W test (verification of normal distribution). Differences between the groups were determined via the Mann-Whitney test, relationships between values of serial (Spearman) correlation coefficients. Differences between occurrence as percentages were calculated using the $\chi^{2}$ test or via the Fisher test (sometimes as the difference between two ratios). Comparison with levels stated by other authors was carried out as a comparison with a reference constant (t-test, STATISTICA program procedure). We consider significance for $\mathrm{p}<0.05$, i.e. a $5 \%$ level of significance.

\section{Results}

In terms of age and anthropometric characteristics there was no statistically significant difference in the groups of women. Anthropometric, metabolic and hormonal characteristics of the group of women with PCOS and the control group are stated in Table 1.

We found statistically significant higher levels of triglycerides in women with PCOS (control average $1.01 \pm 0.7 \mathrm{mmol} . \mathrm{l}^{-1}$, PCOS average $1.17 \pm 0.58 \mathrm{mmol}^{-1}, \mathrm{p}<0.05$ ) (Fig. 1). The two groups did not differ in levels of total, HDL and LDL cholesterol.

In our study we also looked at the relationship between levels of blood lipids and anthropometric indicators in both observed groups. In women with PCOS there was a statistically significant increase in total and LDL cholesterol along with BMI values (positive correlation), in women in the control group this correlation was not proven. In both groups of women we proved a statistically significant relationship between BMI values and levels of triglycerides (positive correlation) (Fig. 2). A negative correlation was proven in the control group between BMI and level of HDL cholesterol, while in women with PCOS this relationship was not proven. In women with PCOS and in the control group there was a statistically significant increase in levels of TAG along with waist measurement, while the level of HDL cholesterol fell. A statistically significant positive correlation between levels of LDL cholesterol and waist measurement was evident only in women with PCOS.

Tab. 1. Monitored anthropometric, metabolic and hormonal parameters and comparison of their statistical significance in a group of women with PCOS and a control group (U test). Results in bold are significant in level $\mathbf{p}<\mathbf{0 . 0 5}$.

\begin{tabular}{|c|c|c|c|}
\hline & $\begin{array}{l}\text { PCOS group } \\
\quad(n=72) \\
\text { Average } \pm \text { SD }\end{array}$ & $\begin{array}{l}\text { Control group } \\
\qquad(\mathrm{n}=43) \\
\text { Average } \pm \text { SD }\end{array}$ & $\mathrm{p}$ \\
\hline Age (years) & $31.6 \pm 7.64$ & $31.4 \pm 7.00$ & 0.991 \\
\hline BMI (kg.m²) & $26.76 \pm 6.08$ & $24.73 \pm 5.66$ & 0.055 \\
\hline Waist (cm) & $84.43 \pm 15.55$ & $79.44 \pm 12.42$ & 0.116 \\
\hline Waist/hip & $0.79 \pm 0.08$ & $0.76 \pm 0.07$ & 0.185 \\
\hline Cholesterol $\left(\mathrm{mmol}^{-1} \mathrm{l}^{-1}\right)$ & $4.76 \pm 0.81$ & $4.68 \pm 0.8$ & 0.608 \\
\hline $\mathrm{LDL}\left(\mathrm{mmol.1} \mathrm{l}^{-1}\right)$ & $2.66 \pm 0.61$ & $2.67 \pm 0.72$ & 0.995 \\
\hline HDL (mmol. $\left.1^{-1}\right)$ & $1.57 \pm 0.33$ & $1.57 \pm 0.4$ & 0.857 \\
\hline TAG $\left(\mathrm{mmol} .1^{-1}\right)$ & $1.17 \pm 0.58$ & $1.01 \pm 0.7$ & 0.021 \\
\hline Glycaemia $\left(\mathrm{mmol.} \mathrm{l}^{-1}\right)$ & $5.04 \pm 0.95$ & $4.91 \pm 0.43$ & 0.802 \\
\hline C peptide (nmol..$\left.^{-1}\right)$ & $0.99 \pm 0.93$ & $0.58 \pm 0.21$ & 0.009 \\
\hline GlyHb (\%) & $3.53 \pm 0.76$ & $3.5 \pm 0.5$ & 0.303 \\
\hline TSH (mU.1-1) & $2.02 \pm 1.49$ & $2.07 \pm 1.61$ & 0.965 \\
\hline fT4 $\left(\right.$ pmol. $\left.1^{-1}\right)$ & $13.61 \pm 2.51$ & $14.67 \pm 2.86$ & 0.043 \\
\hline FSH $\left({\left.\mathrm{U} .1^{-1}\right)}^{-1}\right.$ & $5.95 \pm 3.49$ & $5.05 \pm 2.38$ & 0.240 \\
\hline LH $\left(\mathrm{U} .1^{-1}\right)$ & $8.37 \pm 7.21$ & $6.34 \pm 6.01$ & 0.045 \\
\hline E2 $\left(\right.$ pmol. $\left.1^{-1}\right)$ & $183.91 \pm 112.56$ & $213.4 \pm 219.39$ & 0.424 \\
\hline Progesterone $\left(\mathrm{nmol} . \mathrm{l}^{-1}\right)$ & $4.13 \pm 8.59$ & $9.91 \pm 21.9$ & 0.260 \\
\hline Total test. (nmol.1 $\left.{ }^{-1}\right)$ & $2.68 \pm 1.56$ & $1.43 \pm 1.1$ & 0.000 \\
\hline SHBG $\left(\right.$ nmol. $\left.1^{-1}\right)$ & $59.16 \pm 50.2$ & $62.47 \pm 44.71$ & 0.264 \\
\hline Vol. test. (pg.ml-1) & $5.51 \pm 4.6$ & $1.41 \pm 1.37$ & 0.000 \\
\hline A-dion (ng.ml-1) & $4.79 \pm 3.95$ & $2.5 \pm 1.58$ & 0.000 \\
\hline DHEAS $\left(\mu \mathrm{mol} .1^{-1}\right)$ & $232.13 \pm 149.49$ & $223.96 \pm 144.96$ & 0.993 \\
\hline $17 \mathrm{OH}$ prog. $\left(\right.$ nmol. $\left.1^{-1}\right)$ & $5.46 \pm 4.77$ & $1.64 \pm 1.82$ & 0.000 \\
\hline $\operatorname{PRL}\left(\mathrm{mU} .1^{-1}\right)$ & $381.88 \pm 215$ & $407.55 \pm 325.56$ & 0.421 \\
\hline Hct & $0.41 \pm 0.03$ & $0.41 \pm 0.02$ & 0.615 \\
\hline
\end{tabular}

$\mathrm{SD}$ - standard deviation 


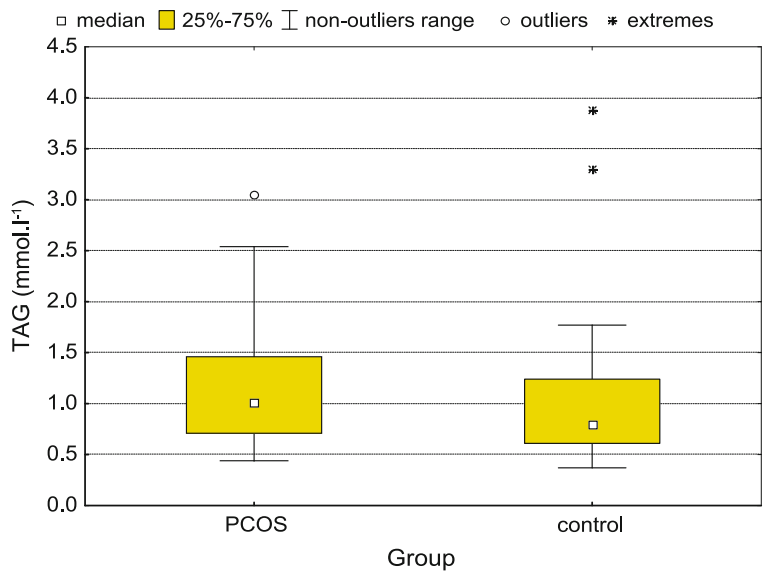

Fig. 1. Levels of triglycerides (TAG) in women with PCOS and control group.

\section{Discussion}

In the question of the level of blood lipids in women with PCOS the results of available studies are inconclusive. Higher levels of triglycerides in women with PCOS have been found in other studies $(6,11,12,14)$. Another study (13) focusing on weight difference between American and Italian women with PCOS also observed levels of blood lipids. American women had pathologically increased levels of triglycerides $\left(1.76 \mathrm{mmol}^{-1} \mathrm{l}^{-1}\right.$; they were distinctly obese women with an average BMI of 40.3 kg. $\mathrm{m}^{-2}$. The level of triglycerides in our women with PCOS (1.17 mmol. $\left.\mathrm{l}^{-1}\right)$ and the Sicilian women in that study $\left(1.03 \mathrm{mmol} . \mathrm{l}^{-1}\right)$ differs statistically significantly $(\mathrm{p}=0.040)$, i.e. in comparison with the Italian women, our women had higher levels despite the fact that they were slimmer. This difference could be due to the different structure of diet in the Czech Republic and Mediterranean region. The role of the diet could be a rather important factor playing a protective role even in overweight and obese women. In the Czech study, the differences in triglyceride levels were found when women with PCOS were divided into patients with or without insulin resistance (IR), namely without IR 1.05 mmol. $1^{-1}$ triglyceride, with IR $1.52 \mathrm{mmol}^{-1}$ (12). In our study we proved a statistically significant relationship between BMI value and visceral obesity characterised by waist measurement and triglyceride level. This discovery corresponds with studies dealing with metabolic syndrome.

Increased levels of triglycerides in women with PCOS cannot be explained simply by obesity and accumulation of visceral fat. Several studies found no difference in TAG even with significant differences in anthropometric characteristics (15).

Inconsistent results also relate to HDL cholesterol - with dyslipidemia combined with metabolic syndrome HDL cholesterol is generally lower. Nevertheless, studies carried out on women with PCOS, including those who are overweight or obese, provide inconclusive results. In our study we did not find any difference in HDL cholesterol levels between the two groups of women. In

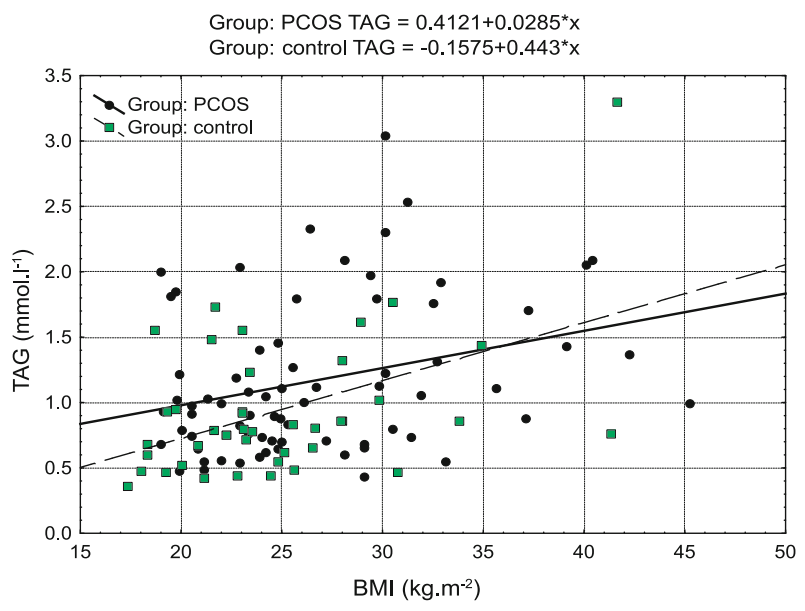

Fig. 2. Correlation graph with regression lines between BMI - body mass index $\left(\mathrm{kg}^{-2} \mathrm{~m}^{-2}\right)$ and triglyceridemia - TAG $\left(\mathrm{mmol} . \mathrm{l}^{-1}\right)$ in women with PCOS $(r=0.304, p<0.05)$ and control group $(r=0.333, p<0.05)$.

women with PCOS there was a statistically significant correlation between HDL cholesterol and waist measurement (negative correlation, as with the control group). Average levels of HDL cholesterol were relatively high $-1.57 \mathrm{mmol}^{-1} \mathrm{l}^{-1}$ in the control group and women with PCOS.

Overall we can state that with increasing BMI the pathologies in blood lipids can be anticipated in women with PCOS, especially in triglyceride levels. Women with PCOS can have surprisingly higher levels of HDL cholesterol than the control group, or they may have comparable levels. When comparing the results of studies it seems that women with PCOS have specific features of metabolic syndrome (16).

Observation of lipoprotein levels as a risk factor of cardiovascular illness in women with PCOS is significant in terms of observing their cardiovascular risk. No distinctly higher incidence of cardiovascular illness has so far been proven in women with PCOS, but few studies have been carried out (14).

\section{Conclusion}

In our study we did not prove any statistically significant differences in anthropometric parameters (BMI, waist measurement, waist/hip ratio) between women with PCOS and a healthy control group of similar age. Lipid metabolism results showed that although the blood triglycerides were within the normal range, a statistically significant difference in their levels was proven between the women with PCOS and control group. The diet could still play a rather underestimated role. Other parameters (total, HDL and LDL cholesterol) did not differ in the two groups.

The importance of changes in blood lipid metabolism in PCOS has been still inconclusive.

Further studies are necessary, especially long-term observation of patients to enable conclusions to be made on the relationship of blood lipid metabolism disorder in women with PCOS and its importance in the risk of cardiovascular illness. 


\section{$569-572$}

\section{References}

1. Chang WY, Azziz R. Pathogenesis of hyperandrogenism in polycystic ovary syndrome. In: Dunaif A, Chang RJ, Franks S, Legro RS. (eds.) Polycystic ovary syndrome: current controversies, from the ovary to the pancreas. Contemporary Endocrinology (Series), Humana Press, Inc. 2008: 281-294.

2. Ehrmann DA. Polycystic ovary syndrome. N Engl J Med 2005; 52 (3): 1223-1236.

3. Azziz R, Carmina E, Dewailly D et al. The Androgen Excess and PCOS Society criteria for the polycystic ovary syndrome: the complete task force report. (Task Force on the Phenotype of the Polycystic Ovary Syndrome of the Androgen Excess and PCOS Society) Fertil Steril 2009; 2: 456-488.

4. ESHRE/ASRM. Revised 2003 consensus on diagnostic criteria and long-term health risks related to polycystic ovary syndrome. Fertil Steril 2004; 81: 19-25.

5. Stein IF, Leventhal ML. Amenorrhea associated with bilateral polycystic ovaries. Am J Obstet Gynecol 1935; 29: 181-191.

6. Apridonidze T, Essah PA, Iuorno MJ, Nestler JE. Prevalence and characteristics of the metabolic syndrome in women with polycystic ovary syndrome. J Clin Endocrinol Metab 2005; 90: 1929-1935.

7. Cibula D. Is insulin resistance an essential component of PCOS? The influence of confounding factors. Hum Reprod 2004; 4: 757-759.

8. Ehrmann DA, Liljenquist DR, Kasza K et al. Prevalence and predictors of the metabolic syndrome in women with polycystic ovary syndrome. J Clin Endocrinol Metab 2006; 91 (1): 48.
9. Cibula D, Cifkova R, Fanta M, Poledne R, Zivny J, Skibová J. Increased risk of non insulin dependent diabetes mellitus, arterial hypertension and coronary artery disease in perimenopausal women with a history of the polycystic ovary syndrome. Hum Reprod 2000; 15: 785-789.

10. Talbott E, Clerici A, Berga SL et al. Adverse lipid and coronary heart disease risk profiles in young women with polycystic ovary syndrome: results of a case-control study. J Clin Epidemiol 1998; 51: 415-422.

11. Pirwany IR, Fleming R, Greer ${ }^{\text {IA et al. }}$ Lipids and lipoprotein subfractions in women with PCOS: relationship to metabolic and endocrine parameters. Clin Endocrinol (Oxf) 2001; 54 (4): 447-453.

12. Vrbikova J, Cifkova R, Jirkovska A, Lanska V, Platilova H, Zamrazil V, Starka L. Cardiovascular risk factors in young Czech females with polycystic ovary syndrome. Hum Reprod 2003; 5: 980-984.

13. Carmina E, Legro RS, Stamets K, Lowell J, Lobo RA. Difference in body weight between American and Italian women with polycystic ovary syndrome: influence of the diet. Hum Reprod 2003; 18: 2289-2293.

14. Landin-Wilhelmsen $K$, Schmidt $M$ et al. Cardiovascular Disease and Risk Factors in PCOS Women of Postmenopausal Age: A 21-Year Controlled Follow-Up Study. JCEM 2011; 96: 3794-3803.

15. Phelan N, O’Connor A, Kyaw-Tun T et al. Lipoprotein Subclass Patterns in Women with Polycystic Ovary Syndrome (PCOS) Compared with Equally Insulin-Resistant Women without PCOS. JCEM 2010; 95 : 3933-3939.

16. Sam S, Dunaif A. Polycystic ovary syndrome: syndrome XX? Trends Endocrinol Metab2003; 14: 365-370. 